\title{
ON-SITE SEMANTIC MAPPING OF ARCHAEOLOGICAL EXCAVATION AREAS
}

\author{
HamidReza Houshiar ${ }^{\mathrm{a}}$, Dorit Borrmann ${ }^{\mathrm{a}}$, Jan Elseberg ${ }^{\mathrm{a}}$, Andreas Nüchter $^{\mathrm{a}, *}$, Falk Näth $^{\mathrm{b}}$, and Stephan Winkler \\ ${ }^{a}$ School of Engineering and Science, Automation Group, Jacobs University of Bremen gGmbH, \\ Campus Ring 1, Bremen 28759, Germany \\ (h.houshiar|d.borrmann|j.elsebergla.nuechter@jacobs-university.de \\ b denkmal3D GbR, Windallee 2, Vechta 49377, Germany \\ falk.naeth@denkmal3. de \\ ${ }^{\mathrm{c}}$ masswerke GbR, Hafenweg 31, Münster 48155, Germany \\ stephan.winkler@masswerke.de
}

KEY WORDS: 3D Laser Scanner, Archeology, Semantic Mapping, 3D Point Cloud Processing

\begin{abstract}
:
3D laser scanning is the state of the art in modeling archaeological excavation sites, historical sites and even entire cities or landscapes. The documentation of findings on an excavation site is an essential archaeological task. Automated systems accelerate this process and decrease the amount of error to a minimum. This paper presents a new documentation approach in industrial archaeology. It consists of a set of tools for recording and registering 3D data from excavation sites. We provide an efficient tool for visualization of acquired 3D point clouds in 3D and 2D modes. The main purpose of this software is to provide an easy to use, on-site semantic mapping tool for archaeologists. It includes functions for selecting and labeling findings. Additional information can be provided for each label. This data is exported to an XML format and serves as input for other systems and databases.
\end{abstract}

\section{INTRODUCTION}

Archaeology is a historical science that is interested in the people and their legacies such as buildings, tools, art, etc. In Germany every large scale construction project is prefaced by a series of inspections. These are performed to determine the possible existence of artefacts or other remains of cultural or historical significance. In order to prevent the destruction of an archaeological site, the building project is accompanied by an archaeologist. It is desirable to excavate efficiently to minimize the cost for the client. This is where the development of our project comes into effect: an easy-to-use hardware and software system allows the archaeologists to digitize the site with 3D laser scanning while digging continuously. This will help archaeological activities to be carried out more efficiently leading to cost saving results. After completion of the archaeological activities, collected data and potential findings will be evaluated and a report will be made available to the authorities.
Until the late 1990s, conventional forms of documentation for archaeological features were based on manual drawings on graph paper. These sketches provide levels, colors and numbers for findings with a connection to a detailed catalog. Findings are also photographically recorded within these documents. In this way, the digging process is meticulously recorded during the excavation. Figure 1 (left) shows an excavation site in Münster, Germany in May 2011. The right part of the figure shows a map of findings on graph paper. The aim is to document the findings in retrospect as precisely as possible and describe them in three dimensions based on their chronological sequence. Documents are evaluated after completion of the excavation. The findings provide insights on early culture, religion, handcraft, daily life and social process on both local and national levels.

Technological achievements in the last 10 years enable higher accuracies in the documentation process. Moreover they could accelerate this process with regard to economic constraints. But, they create at the same time a large amount of data that is no
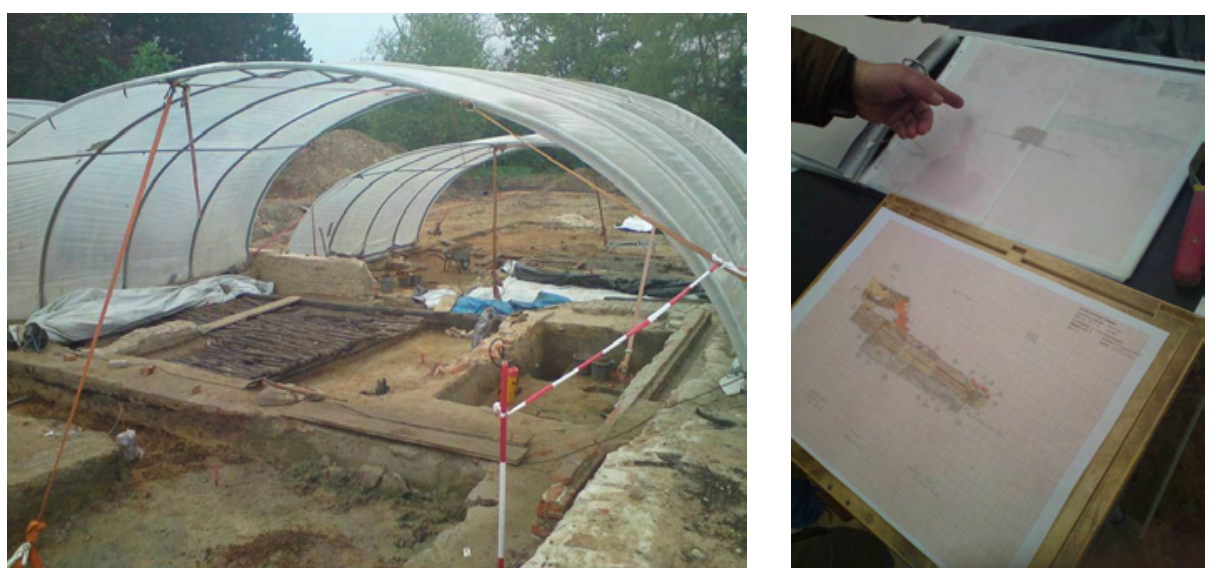

Figure 1: Left: Overview of an excavation site located at "Haus Kump", a building from the 16th century, which is the oldest storage building in Münster, Germany. Right: Findings on a graph paper that were meticulously sketched by an archeologist on site. 
longer subject to the evaluation judgment of the archaeologist. Automatic acquisition of data occurs even without interaction of the archaeologist.

Apart from photogrammetry 3D laser scanners are also in use in archeology. The 3D scans taken from archaeological sites often only serve as snapshots or as as-built documentation and reference. Campana and Remondino (2007) remark in their publication that "The balance of the load decades of archaeological research in the use of 3D digital documentation/representation in terms of scientific investigation is quite disappointing if we think at the possibility offered nowadays by newly developed software and hardware. The use of 3D images was typically oriented to suggest final reconstructions and not to contribute to scientific interpretation.".

3D laser scanners are becoming the standard instrument in the field of optical metrology. They send a focused laser beam in one direction and determine the distance to a point on a surface. The distance is calculated by measuring the time difference of the emitted and reflected signals. Two different types of laser scanning technology have established themselves in this field. Pulsed laser range finders send a short pulse of light and directly measure the amount of time it takes for the laser pulse to travel from the scanner to the object and back to the scanner. The distance is then calculated via the speed of light. Since the speed of light is almost $300000 \mathrm{~km} / \mathrm{s}$, tools with picoseconds $\left(10^{-12} \mathrm{~s}\right)$ resolution need to be used in order to calculate the distance with an accuracy of $10 \mathrm{~mm}$. Other than pulsed systems there are laser scanners that continuously emit modulated laser light. The change of the phase between the emitted light and the received signal determines the light travel time. Since the phase shift in only one interval between 0 and $2 \pi$ is clear, the maximum range of these devices is inherently limited. Current phase-based laser scanners achieve a range of about $80-120 \mathrm{~m}$ whereas pulse-based laser can measure distances up to several $\mathrm{km}$. However, since phase-based laser scanners emit light continuously they can operate at higher measurement speed than pulsed systems. Besides the distance to objects, laser scanners determine the intensity of the reflected light. This yields information about the reflectivity of the scanned surface. Further combination of the Light Detection and Ranging (LiDAR) systems with color cameras is possible.

The paper presents a software tool for archeologists that can be used to acompany excavations. It is built based on $3 D T K-T h e$ $3 D$ Toolkit. 3D laser scans obtained by frequently scanning the archeology site are registered onto a common coordinate system and efficiently visualized to the archaeologist on a tablet computer. Findings are marked on-site with a closed polygon and this localized information is send to an archeologal database for documentation and reasoning.

\section{RELATED WORK}

Cultural sites have an inestimable value. However, often they have only been recognized after they are damaged or destroyed. With the use of technology and 3D modeling techniques it is possible to reconstruct such cultural heritage objects. Wüst et al. (2004) propose applying the 3D GIS project DILAS (Digital Landscape Server) to archaeology and cultural heritage projects. The original aim of DILAS was the efficient generation, management and visualization of large 3D landscapes and city models. Karmacharya et al. (2008) suggest the use of a web platform based on knowledge management and semantic web technologies. It is used to store the data during the excavation process and to manage the knowledge acquired during the identification process of findings. Since different technologies are being used during the excavation, different patterns of data are generated. One of the sources of data are the 3D point clouds obtained through the terrestrial laser scanning process. Point clouds, floor plans, images and archaeological notes are collected during the project for the creation of 3D object models. Non-academic archaeology generates a huge amount of data in a very short period of time. Thus, the collected data is stored in a repository. Once the data is stored, the process of identification of findings is carried out. Cosmas et al. (2001) introduce the "3D Measurement and Virtual Reconstruction of Ancient Lost World of Europe" system (3D MURALE). This system consists of a set of tools for recording, reconstructing, encoding, visualizing and database searching or querying. These tools are linked together by a common database. The database serves several purposes. It contains information about the pieces. It also serves as a repository for archaeologist to help them to classify findings, to prepare restoration and to keep track of statistics. The database is a major gateway to the wider public and to other archaeologists. Grabczewski et al. (2001) describe the 3D MURALE multimedia database system architecture. The goal of this system is to digitally record, store, restore and visualize archaeological findings.

Semantic perception, mapping and exploration for intelligent systems has seen a lot of progress recently, especially in the computer vision and robotics community (Nüchter et al., 2013 , in press). Segmentation and annotation of natural scenes, e.g., from images or point clouds is one of the topics in semantic perception and mapping. Semantics is the study of meaning and hence semantic perception focuses on interpreting and organizing sensor information in a symbolic form. Semantic mapping combines the extration of semantics with mapping. Actually there is no map without the meaning. While a map can be two dimensional or three dimensional, it always stores either raw sensor data, like 3D point clouds, or syntactic elements, like lines or 3D planes, or it stores semantic features, like interpreted objects. Semantic perception, mapping and exploration is also related to scene understanding, cognitive vision, object detection, object recognition and sensor data processing in general.

Laser scanners are state of the art in modeling archaeological and historical sites. Digitizing an excavation site without occlusions requires multiple $3 \mathrm{D}$ scans, i.e., $3 \mathrm{D}$ point clouds. To create a correct and rigid model, the scans have to be merged into one coordinate system. This process is called scan registration. A popular algorithm to automatically merge two independently acquired scans was already proposed in 1991. This algorithm is called iterative closest point algorithm (ICP) (Besl and McKay, 1992). Most of recent terrestrial laser scanners provide reflectance and color data in addition to range information. Panorama images can be generated from these point clouds. Image generation from point clouds will include the conventional $2 \mathrm{D}$ image matching algorithms to point clouds. Böhm and Becker (2007) explore the application of scale invariant feature transform (SIFT) method on laser reflectance data. In our previous work we study the different projections for key point based registration of panoramic terrestrial 3D laser scans (Houshiar et al., 2013). Nüchter et al. (2011) present an approach based on the existence of a skyline, i.e., a border between buildings and sky. These features are extracted from 3D panoramic images.

\section{3D SCAN REGISTRATION}

Digitizing environments without occlusions requires multiple 3D scans, i.e., 3D point clouds. Therefore, the creation of a rigid 3D model from several point clouds requires scan registration. The procedure can be formulated as follows: Acquire a series of 3D laser scan data, find initial feature or point correspondences 


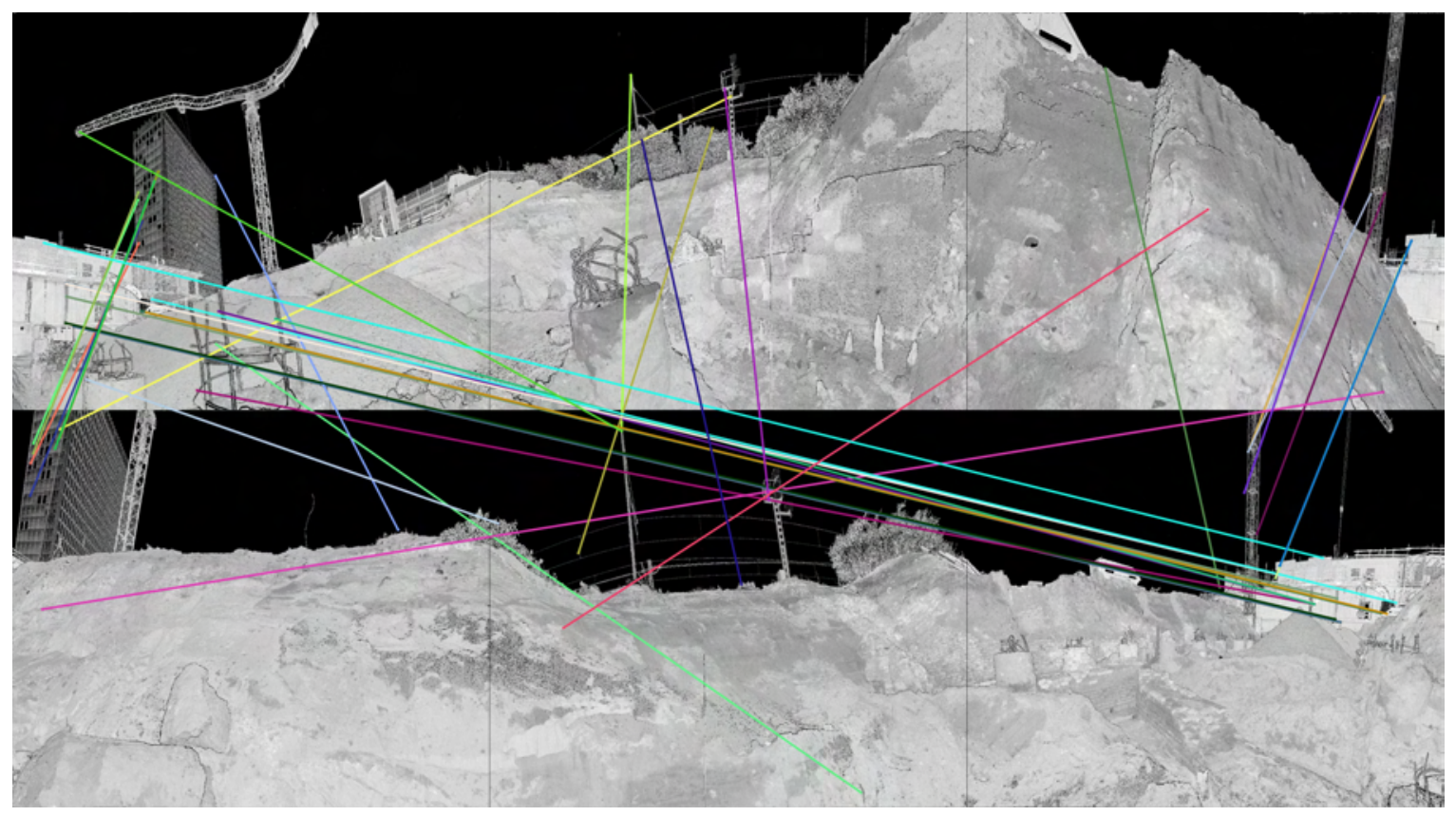

Figure 2: Registration of two 3D scans acquired at an excavation site using SIFT features and SIFT descriptors on a Pannini based projection of the full 360 degree scan. For the Pannini projection, the image was divided into three parts.

for every two scan pairs, and compute the relative orientation between scans.

\subsection{Feature-based Registration}

The computation of the transformation matrix is based on the correspondences in scan pairs. Many state of the art registration methods rely on initial pose (position and orientation) estimates, global positioning system (GPS) or local positioning using artificial landmarks or markers as reference (Wang et al., 2008). Other methods rely on natural features. These features are generated automatically from the 3D scans.

3.1.1 Markers for registration Marker based registration uses defined artificial landmarks as corresponding points. This supervised data association ensures that the scans are registered at their correct location. Marker based algorithms manually or automatically extract the 3D position of the markers for registration. The registration with the installed markers is precise. However, these algorithms require the manual installation of markers on the scan site. This increases the time of the workflow and requires more interaction.

3.1.2 Registration using natural features Automatic algorithms favor the automatic extraction of natural features from scans, which are then exploited to find the correspondences of scan pairs. Lee et al. (2004) present a matching method in order to find the correspondences of features in two omnidirectional images. Hansen et al. (2007a,b) consider an application of scale invariant feature detection using scale-space analysis suitable for use with wide field of view cameras.

The reflectance intensity provided by modern laser scanners gives additional information for registration. Böhm and Becker (2007) suggest the use of SIFT features for automatic registration. Weinmann et al. (2011); Weinmann and Jutzi (2011) present a method that is based on both reflectance and range images. All these approaches find the correspondences for registration based on the features extracted from the images and directly from the point cloud.
Other approaches rely on the 3D structure. Brenner et al. (2008) use 3D planar patches and the normal distribution transform (NDT) on several 2D scan slices for registration. These approaches use the global features of the scans. Several researchers use features that describe small regions of the scan for place recognition and registration of scans (Huber, 2002; Steder et al., 2010; Barnea and Filin, 2008).

Our registration approach is to convert 3D scans into panorama images, extract features from these images, use feature descriptors for data association and compute the transform using RANSAC and a least square method (Houshiar et al., 2013). An example is given in Figure 2.

\subsection{Point-based Registration}

Point-based algorithms require no computation of features. Instead, they match point clouds by selecting point correspondences Iterative closest point (ICP) is a well known algorithm for pointbased registration (Besl and McKay, 1992). It works by point correspondences on the basis of smallest distance and minimizing the resulting Euclidean error. This iterative algorithm converges to a local minimum. Good start estimates improve the matching result by ensuring the convergence to a correct minimum. Start estimates can be generated from feature based algorithms and this algorithm can be used for fine registration.

\subsection{Global Relaxation}

Registration of 3D point cloud pairs using mentioned algorithms is prone to errors. Errors accumulate in the registration of several 3D data sets. These errors are due to imprecise measurements and small registration errors. Globally consistent scan matching algorithms aim at reducing these errors. To avoid these problems, global matching algorithms are required, taking correspondences from all scans into account. Lu and Milios (1997) presented a solution using a network of relations between laser scan poses. A single linear equation system yields optimal estimations for all poses. Their approach is limited to 2D laser scans. Borrmann et al. (2008) extend the linear estimation algorithm to work reliably with 3D scans and 6 Degrees of Freedom. They have presented a 


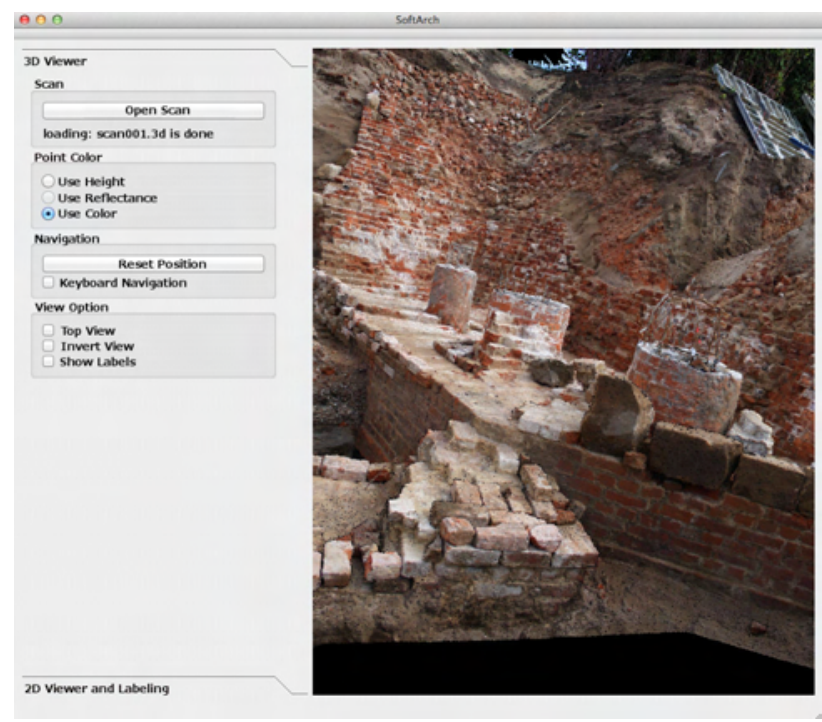

Figure 3: 3D viewer of Castle3D software. Presenting an excavation site in Bremen, Germany.

technique of matching laser scans achieving global consistency. They avoid the common problems of sequential matching strategies by minimizing a global error function in a bundle adjustment like manner.

\section{SEMANTIC LABELING}

\subsection{Castle3D for on-site labeling}

Data management is widely used in archaeological projects to store and retrieve generated information during and after an excavation. Rapid growth of technology makes it possible to produce huge amounts of data in a short period of time. Therefore management of data has become problematic with conventional methods. The primary motivation of computer aided systems in archaeology is to produce new ways of recording, cataloging, encoding and visualizing archaeological artifacts and monuments. $3 \mathrm{D}$ technologies have produced portable and fast systems to record large amounts of precise 3D data efficiently. Our system is an additional tool for archaeologist to visualize and register point clouds that have been acquired from excavation sites. The registration of scans and the on-site visualization helps during the process of removing occlusions. Recently Elseberg et al. (2013) have introduced a fast and easy to use visualization tool that visualizes point clouds with millions of points in a matter of milli seconds (Figure 3). The visualization tool has improved the interaction of archaeologist with the recorded data of an excavation site. Non-academic archaeology sites are often only available for a very short period of time. Therefore, the excavation process has to happen quickly. Recording data in 3D with laser scanners will provide detailed data of the site. Reconstruction and visualization of the data after the actual excavation assists archaeologist in studying the site more precisely. It also produces an infrastructure for other archaeologists to study the excavation sites based on their own knowledge.

Currently, archaeological observations are recorded on-site using conventional methods (cf. Fig. 1). The outlines of findings are drawn on graph paper with detailed information based on subjective understanding of the findings. Images, floor plans and other data such as archaeological notes are collected during the project. These data are of great value in the analysis of archaeological findings in any project. 3D data acquisition and computer aided system will facilitate this process and they are less prone to human error.

In our software, called Castle3D (Computer aided system for labeling archeological excavations in $\underline{3 \mathrm{D}}$ ) a series of labeling tools assist archaeologist to document their findings on-site for further inspections. We have implemented a $2 \mathrm{D}$ representation of the $3 \mathrm{D}$ data. This representation facilitates the selection process for regions of interest. Afterwards, the selected region of interest is available in both $2 \mathrm{D}$ and $3 \mathrm{D}$ representations of the scan (see Figure 4). A series of predefined categories are available to document the labels. Additional notes can be added to each label. This computer system facilitates the identification process of the findings on-site. They also provide feasible tools to study and observe the excavation site and findings both for the archaeologist on the site and for the public. To enable the combination of several laser scans the registration methods described in the previous section have been implemented in the software.

\subsection{Data Structures for Efficient 3D Point Cloud Data Pro- cessing}

Modern terrestrial scan systems acquire data at an impressive rate. To load and process large point clouds in the main memory on a standard system we use two efficient data structures for 3D point clouds: an octree and a panorama image array.

In (Elseberg et al., 2013) we describe a spatial data structure called octree with a low memory consumption. An octree is a tree data structure that is used to index 3D data. Each node of the octree represents the volume formed by a rectangular cuboid. An octree node has up to eight children. Each child corresponds to one octant of the cube. A node without a child implies that for the corresponding volume no further subdivision is necessary and the volume can be uniformly represented. When storing a point cloud, they defined a stopping rule for occupied volumes. The stopping criteria is defined as both maximal depth and minimal number of points. This criteria is applied to volumes without points, such that child nodes are created only for volumes that contain points. All nodes without children are considered as empty space. As laser scanners sample the surface of objects, the acquired 3D point clouds that is not fully volumetric. Since most space in point clouds is not occupied, most octree nodes will only have few children. The octree data structure is therefore ideally suited for 3D laser scanner data. Figure 5 shows a recursive refinement of an octree.

Most modern laser scanners provide additionally color and reflectance information to range information. This information has been used by numerous applications to produce images. The 2D representation of a $3 \mathrm{D}$ point cloud permits the use of conventional $2 \mathrm{D}$ algorithms for acquired $3 \mathrm{D}$ data. We use the range information to produce panorama images of scans (cf. Fig. 5). All pixels of the image contain color, intensity and a list of $3 \mathrm{D}$ points, that fall into the array element. A panorama image offers a full view of the excavation site in one image and provides a better understanding of the environment. Archaeologists are able to mark the findings in panorama images and provide additional information for each of the findings. The process of labeling the findings is facilitated by a set of marking tools. These tools will distinguish the selected region of interest based on predefined categories. Additionally, these regions of interest can be segmented from the rest of the point cloud. The segmented region will represent findings, such as monuments, buildings, stones, and other structures. Figure 4 (right) illustrates a panorama image with a marked region of interest presented in the 2D viewer of the Castle3D software. The $2 \mathrm{D}$ view is based on generated panorama images from the input 

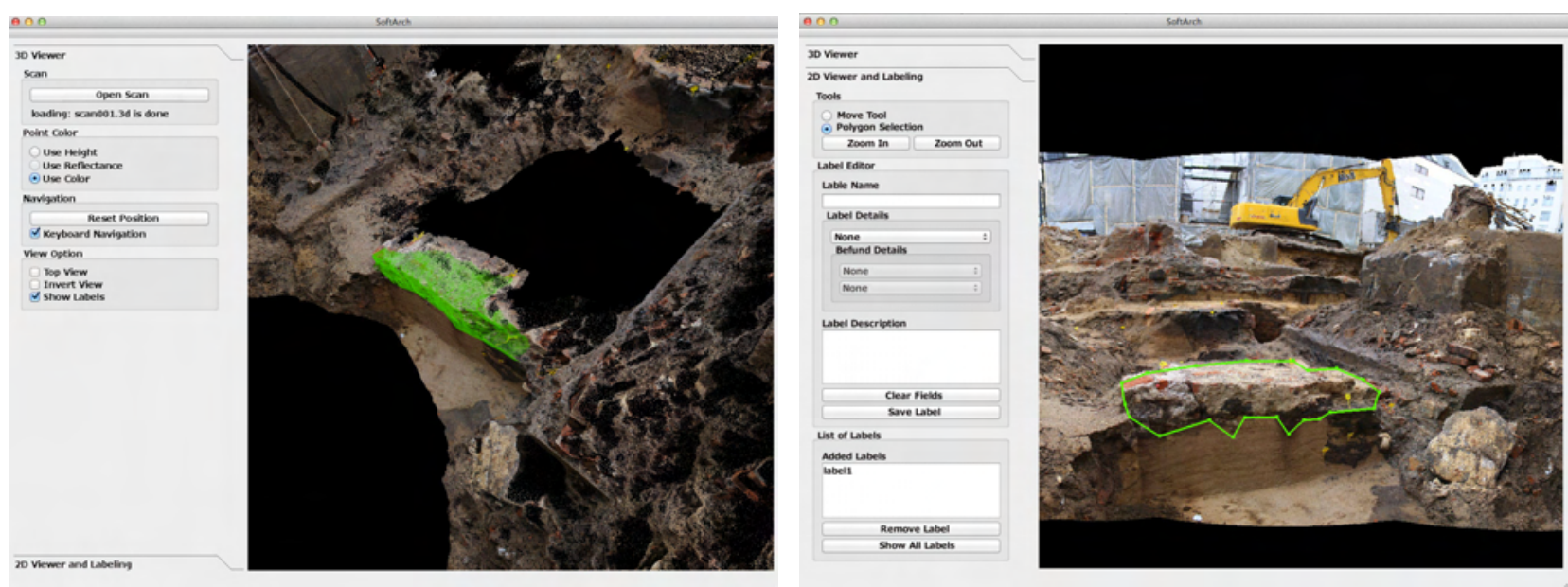

Figure 4: A presentation of a selected region of interest of a scan in the 3D viewer (left) and in the 2D viewer of the Castle3D software. The scan has been taken at an excavation site in Bremen, Germany.
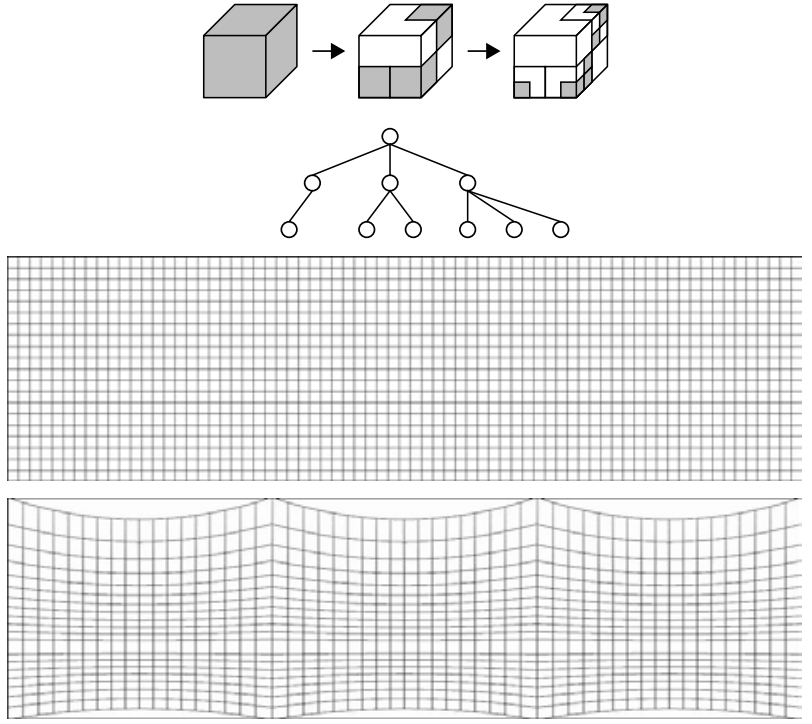

Figure 5: Top: Spatial subdivisions of an octree up to level 3. Occupied leaf nodes are shaded gray. The corresponding tree structure of the sparse data structure. Middle: Panorama image in eqirectangular prjection. Bottom: Panorama image composed of 3 Pannini projections as used in Fig. 2.

scan. Tools for region selection and documentation are provided in this view of the software.

Documentation is essential to archaeological analysis. Therefore, it is crucial to have a proper amount of documentation for each finding. These data are based on the archaeologist's understanding and experience. Since the data have been acquired by terrestrial laser scanners and represented by computer aided systems, they can be studied in the archaeology community. The data can be used for further studies, catalogues, archaeological databases, and archaeological data management systems. We offer an Extensible Markup Language (XML) file format to export the data and documentation of findings. This is essential for multi-platform communication. The XML file can be easily imported into other software.

\subsection{Constraint Checking}

Methods are available to verify the interpretation of the findings on site. Harris matrix is a tool to represent the stratigraphic units and the relations between different layers and findings (Harris,
1989). The matrix reflects the relative position and stratigraphic contacts of observable stratigraphic units. In general, one can assume that the recent layers lie above the rest of the layers and the earliest at the bottom. This is constructed by representing the connection of these layers. The lines represent the direct stratigraphic contact. This pie graph representation of layers assists in informing the archaeologist on the physical process of site formation and to highlight areas where loops in recorded sequence occur. These loops in the matrix produce temporal anomalies. These anomalies present that in a sequence of context, the earliest in the sequence appears to be later than the latest context. This happens by virtue of errors in excavation or recording. During urban excavations, thousands of results will be produced, so the on site consistancy check is vital.

Regular, e.g., daily scans of the excavation site help the compilation of the Harris matrix. This matrix can be generated automatically or semi-automatically with the help of an archaeologist. Our system provides a tool for the compilation of the matrix as it uses the database Adiuvabit (maßwerke GbR, 2008) as back end. Moreover, the matrix can be generated after the excavation from the original data. This improves the objectivity of the analysis of the findings. Excavation sites can be studied by the archaeology community and they can compile a new Harris matrix based on their understanding from the original scans.

\subsection{Experimental Data Set}

All figures in the paper have been generated with our software. Throughout the paper, we have used data from two excavation sites. Fig. 2 originates from the remains of the Stephanitorzwinger (Bräutigam), a famous city tower of Bremen storing black gun powder that was destroyed in 1647 by a stroke of lightning. It was discovered last year during the construction of a theatre. The remaining figures have been rendered from the Motel One construction site in Bremen downtown. Animations through both 3D point clouds are given at our youtube channel "AutomationAtJacobs" under http://youtu.be/XZiji3q-wGg and http:// youtu.be/1-PSBOUmZZE .

\section{CONCLUSIONS}

We have presented an easy to use, on-site semantic mapping tool for archaeology excavation sites. The 3D scans taken from excavations are used to automate the documentation process. This project contains a $3 \mathrm{D}$ viewer that presents the acquired 3D data. 
Non-academic archaeological sites are only accessible for a very short period of time. Therefore, automated systems can increase the speed of the digging. Conventional methods are based on graph paper and are prone to human perception and errors. The presented software assists archaeologists in decreasing these errors while improving the efficacy of the excavation. The 2D viewer and labeling system presented in this project helps the annotation and analysis process. The system provides the means to study the excavation site after a period of time.

\section{ACKNOWLEDGMENTS}

This work was funded by the project "Fortlaufende semantische 3D-Kartierung von archäologischen Ausgrabungsstätten" by the German Federal Ministry of Economics and Technology by a Central Innovation Program (ZIM; No. KF24700098HM2) due to a resolution of the German Bundestag.

\section{References}

Barnea, S. and Filin, S., 2008. Keypoint based autonomous registration of terrestrial laser point-clouds. ISPRS Journal of Photogrammetry and Remote Sensing 63(1), pp. 19-35.

Besl, P. and McKay, N., 1992. A method for registration of 3-d shapes. IEEE Transactions on Pattern Analysis and Machine Intelligence (PAMI) 14(2), pp. 239 - 256.

Böhm, J. and Becker, S., 2007. Automatic marker-free registration of terrestrial laser scans using reflectance features. In: Proceedings of 8th Conference on Optical 3D Measurement Techniques, Zurich, Switzerland, pp. 338-344.

Borrmann, D., Elseberg, J., Lingemann, K., Nüchter, A. and Hertzberg, J., 2008. Globally consistent 3D mapping with scan matching. Journal of Robotics and Autonomous Systems (JRAS) 56(2), pp. 130-142.

Brenner, C., Dold, C. and Ripperda, N., 2008. Coarse orientation of terrestrial laser scans in urban environments. ISPRS Journal of Photogrammetry and Remote Sensing 63(1), pp. 4-18.

Campana, S. and Remondino, F., 2007. Fast and detailed digital documentation of archaeological excavations and heritage artifacts. In: Proceedings of 35th CAA Conference (Computer Applications and Quantitative Methods in Archaeology), Berlin, Germany, pp. 36-42.

Cosmas, J., Itagaki, T., Green, D., Grabczewski, E., Weimer, F., Gool, L. J. V., Zalesny, A., Vanrintel, D., Leberl, F., Grabner, M., Schindler, K., Karner, K. F., Gervautz, M., Hynst, S., Waelkens, M., Pollefeys, M., DeGeest, R., Sablatnig, R. and Kampel, M., 2001. 3D murale: A multimedia system for archaeology. In: Virtual Reality, Archeology, and Cultural Heritage, pp. 297-306.

Elseberg, J., Borrmann, D. and Nüchter, A., 2013. One billion points in the cloud - an octree for efficient processing of 3D laser scans. ISPRS Journal of Photogrammetry and Remote Sensing (JPRS) 76, pp. 76-88.

Grabczewski, E., Cosmas, J., Santen, P. V., Green, D., Itagaki, T. and Weimer, F., 2001. 3D murale: multimedia database system architecture. In: Virtual Reality, Archeology, and Cultural Heritage, pp. 315-322.
Hansen, P., Corke, P., Boles, W. W. and Daniilidis, K., 2007a. Scale-invariant features on the sphere. In: ICCV.

Hansen, P., Croke, P., Boles, W. and Daniilidis, K., 2007b. Scale invariant feature matching with wide angle images. In: In IEEE/RSJ International Conference on Intelligent Robots and Systems.

Harris, E. C., 1989. Principles of Archaeological Stratigraphy. Second edition edn, Academic Press, Londod \& New York.

Houshiar, H., Elseberg, J., Borrmann, D. and Nüchter, A., 2013. A study of projections for key point based registration of panoramic terrestrial 3D laser scans. Geo-spatial Information Science (GSIS).

Huber, D., 2002. Automatic Three-dimensional Modeling from Reality. PhD thesis, Carnegie Mellon University.

Karmacharya, A., Cruz, C., Marzani, F. and Boochs, F., 2008. Industrial archaeology: Case study of knowledge management for spatial data of findings. In: 5th International Conference on Adaptive Hypermedia and Adaptive Web-Based Systems.

Lee, Y. J., Kim, D.-Y. and Chung, M. J., 2004. Feature matching in omnidirectional images with a large sensor motion for map generation of a mobile robot. Pattern Recogn. Lett. 25(4), pp. $413-427$.

Lu, F. and Milios, E., 1997. Globally consistent range scan alignment for environment mapping. Autonomous Robots 4, pp. $333-349$.

maßwerke GbR, 2008. Adiuvabit. http://www adiuvabit. de.

Nüchter, A., Gutev, S., Borrmann, D. and Elseberg, J., 2011. Skyline-based registration of 3D laser scans. Geo-spatial Information Science 14(2), pp. 85-90.

Nüchter, A., Rusu, R. B., Holz, D. and Munoz, D., 2013 (in press). Editorial: Semantic perception, mapping and exploration. Journal of Robotics and Autonomous Systems (JRAS).

Steder, B., Grisetti, G. and Burgard, W., 2010. Robust place recognition for $3 \mathrm{D}$ range data based on point features. In: Proceedings of the IEEE International Conference on Robotics and Automation (ICRA '10), pp. 1400-1405.

Wang, X., Toth, C., Grejner-Brzezinska, D. and Sun, H., 2008. Integration of terrestrial laser scanner for ground navigation in gps-challenged environments. In: Proceedings of the XXIst ISPRS Congress: Commission V, WG 3, pp. 513-518.

Weinmann, M. and Jutzi, B., 2011. Fully automatic image-based registration of unorganized tls data. In: ISPRS Workshop Laser scanning 2011, ISPRS.

Weinmann, M., Weinmann, M., Hinz, S. and Jutzi, B., 2011. Fast and automatic image-based registration of tls data. ISPRS Journal of Photogrammetry and Remote Sensing 66(6, Supplement), pp. 62-70.

Wüst, T., Nebiker, S. and Landolt, R., 2004. Applying the 3D gis dilas to archaeology and cultural heritage projects - requirements and first results. In: O. Altan (ed.), International Archives of Photogrammetry Remote Sensing and Spatial Information Sciences, Vol. 35, pp. $407-412$. 